\title{
Acceso cubital primario para realizar estudios angiográficos coronarios en 60 pacientes
}

\author{
Primary ulnar access to study coronary angiography in 60 patients
}

Iván Ortega C., Hernando Benítez D., Racid Carmona B., David Truchi, Marcelo Bettinotti' ${ }^{1}$, Carlos Sztejfman, Francisco Garrido

\section{RESUMEN}

Objetivo. Determinar la factibilidad y seguridad de realizar los procedimientos angiográficos coronarios por acceso cubital como vía de elección primaria.

Material y método. Se evaluó en forma prospectiva, multicéntrica, observacional no aleatorizada a los pacientes en los que se eligió el abordaje cubital como acceso primario para procedimientos diagnósticos y terapéuticos en los servicios de hemodinamia de clínica Mar Caribe, clínica CEHOCA, instituto CENCAR, ubicados en la ciudad de Santa Marta (Colombia), entre el 15 de enero de 2018 y el 15 de enero de 2019. Resultados. De un total 1020 estudios practicados, se utilizó la vía cubital de forma primaria en 60 casos (5,8\%). Los pacientes hipertensos correspondían al 91\%, los diabéticos al $35 \%$, los tabaquistas al $35 \%$. Se practicaron procedimientos diagnósticos a $57(95 \%)$, a 2 (3,3\%) solo un procedimiento terapéutico programado y en uno $(1,6 \%)$ la punción no fue exitosa. En 14 pacientes se realizó angioplastia (23\%), de los cuales en 5 (9\%) la angioplastia fue primaria y en 9 (14\%) fue electiva. A 20 pacientes (33\%) se les realizó más de un procedimiento por la misma vía durante el estudio. Se obtuvo una tasa de éxito del $100 \%$ y $0 \%$ de mortalidad en las angioplastias, y $97 \%$ de éxito en los diagnósticos. No se presentaron complicaciones isquémicas, un paciente presentó hematomas grado I. No se registraron complicaciones clínicas hasta el alta. Conclusión. De acuerdo a los resultados obtenidos, el acceso cubital como vía primaria es factible y seguro para la realización de estudios coronarios ya sean diagnósticos o terapéuticos coronarios, además se puede realizar otros tipos de estudios angiográficos y múltiples procedimientos durante el mismo acto con excelentes tasas de éxito, bajas tasas de complicaciones, independientemente del contexto clínico del paciente.

Palabras claves: acceso cubital, arteriografía coronaria.

\begin{abstract}
Objective. To determine the feasibility and safety of performing coronary angiography procedures for ulnar access as the primary route of choice.

Material and Method: The patients in whom the ulnar approach was chosen as the primary access for diagnostic and therapeutic procedures in the hemodynamic services of Mar Caribe clinic, CEHOCA clinic, CENCAR institute, were evaluated prospectively, multicenter in a non-randomized observational manner. located in the city of Santa Marta (Colombia), between January 15, 2018 and January 15, 2019.

Results. Of a total of 1020 studies, the ulnar route was used in 60 cases of primary form (5.8\%). Hypertensive patients (pct) corresponded to $91 \%$, diabetics $35 \%$, tobacco $35 \%$. Diagnostic procedures were performed at 57 pct (95\%), in 2 pct (3.3\%) only the programmed therapeutic procedure was performed and at 1 pct $(1.6 \%)$, the puncture was not successful. 14 pct were subjected to angioplasty (23\%), of these primary angioplasties was performed in 5 pct (9\%) and 9 pct (14\%) were performed elective angioplasty. At 20 pct (33\%) more than one procedure was performed in the same way during the study. The success rate was $100 \%$ and $0 \%$ mortality in angioplasty and $97 \%$ success in diagnoses. There were no ischemic complications, one pct presented grade I hematomas. There were no clinical complications until discharge.

Conclusion. According to the results obtained, the ulnar access as a primary route is feasible and safe for the performance of coronary studies, whether diagnostic or coronary therapeutic, in addition other types of angiographic studies and multiple procedures can be performed during the same act with excellent rates of success, low rates of complications, independent of the clinical response of the patient.
\end{abstract}

Key words: ulnar access, coronary arteriography.

Revista Argentina de Cardioangiología Intervencionista 2019;10(1):13-16. DOl: 10.30567/RACI/201901/0013-0016

\section{INTRODUCCIÓN}

El acceso por vía radial para procedimientos diagnósticos y terapéuticos coronarios se ha transformado en la vía primaria en muchos centros del mundo, dada la baja tasa de complicaciones vasculares, la comodidad y confort para el paciente y la posibilidad de deambulación precoz que se traducen en menor estadía hospitalaria. Sin embargo, las variantes anatómicas, de diámetro, así como la inadecuada irrigación colateral o el vasoespasmo, no siempre hacen posible el acceso por esta vía para los procedimientos ${ }^{1,2}$.

Hasta la realización de este estudio, el acceso radial es la vía de elección primaria en nuestros centros. Se elige como vía alternativa el acceso femoral y en casos seleccionados la vía humeral, quedando el acceso cubital como última alternativa para realizar estudios angiográficos coronarios.

Desde el año 2001 se encuentran estudios en los cuales se utiliza la vía cubital como alternativa para realizar estudios angiográficos coronarios, como lo reportaron Terashima et al. ${ }^{3}$ Posteriormente se incluye la vía cubital como alternativa

1. Servicios de Hemodinamia de Clínica Mar Caribe, clínica CEHOCA e instituto CENCAR, ubicados en la ciudad de Santa Marta, Colombia

$\triangle$ Correspondencia:Dr.Iván Ortega Carrascal.ivanortega05@hotmail.com Los autores no declaran conflictos de intereses

Recibido: 31/01/2019| Aceptado: 14/03/2019 viable para realizar estudios coronarios cuando el acceso por vía radial no fue exitoso ${ }^{4}$. Lo que transformó este acceso en una alternativa viable en pacientes seleccionados, aun cuando las características anatómicas (principalmente la cercanía con el nervio cubital y su profundidad) la hacen potencialmente propensa a complicaciones diferentes de las de la arteria radial, lo que a su vez frenó el empleo de este acceso como vía habitual en los servicios de Hemodinamia. En nuestra búsqueda se encontraron estudios con más de 400 pacientes que compararon el acceso cubital como alternativa a la vía radial demostrando la efectividad y seguridad de esta con tasas de éxito similares ${ }^{4-6}$.

\section{MATERIAL Y MÉTODO}

Se evaluó en forma prospectiva, multicéntrica, observacional no aleatorizada a pacientes en quienes se eligió el abordaje cubital como acceso primario para procedimientos diagnósticos y terapéuticos en los servicios de Hemodinamia de clínica Mar Caribe, clínica CEHOCA, instituto CENCAR, ubicados en la ciudad de Santa Marta (Colombia), entre el 15 de enero de 2018 y el 15 de enero de 2019.

\section{OBJETIVO}

Determinar la factibilidad y seguridad de realizar los procedimientos angiográficos coronarios por acceso cubital como 
TABLA 1. Evaluación del pulso.

\begin{tabular}{|l|}
\hline Pulsos periféricos \\
\hline 0 Pulso no palpable \\
\hline 1+ Pulso palpable, pero fácilmente obliterado, débil, filiforme. \\
\hline 2+ Pulso débil, pero no puede obliterarse \\
\hline 3+ Fácil de palpar, lleno, no puede obliterarse \\
\hline 4+ Fuerte, pulso intenso, puede ser anormal. \\
\hline
\end{tabular}

TABLA 2. Definición de hematoma.

\begin{tabular}{|c|l|}
\hline Grado & Definición \\
\hline I & Superficial, de menos de $5 \mathrm{~cm}$ del sitio de punción \\
\hline II & Moderada infiltración muscular, de menos de $10 \mathrm{~cm}$ del sitio de punción \\
\hline III & Antebrazo con infiltración muscular que llega al codo \\
\hline IV & Infiltración muscular que se extiende proximal al codo \\
\hline V & Síndrome compartimental \\
\hline
\end{tabular}

vía de elección primaria, en una serie de 60 casos seleccionados de forma consecutiva.

\section{DEFINICIONES}

1. Infarto de miocardio: evento coronario agudo con síntomas de isquemia miocárdica y evidencia electrocardiográfica, analítica (biomarcadores de necrosis) o patológica de infarto según la definición AHA/ACC.

2. Angioplastia electiva: la que se realiza en un paciente compensad medicamente y sin compromiso Hemodinámico al momento del Estudio.

3. Angioplastia de urgencia: corresponde a cualquiera de las siguientes condiciones: ATC primaria, la que es realizada en las primeras 12 horas de IAM con supradesnivel del ST; ATC de emergencia, la realizada en un paciente que no logra ser compensado medicamente.

4. Pulso adecuado: presencia de pulso palpable bilateral no obliterable.

5. Cross over: requerimiento de acceder por vía alterna a la establecida de forma primaria por la siguiente causa: punción prolongada, más de 2 intentos de punción máximo 4 intentos para acceder a la vía elegida; alteraciones anatómicas, variantes anatómicas arteriales que impidan completar el estudio.

6. Hematoma: presencia de infiltrado sanguíneo en piel cuyo grado dependerá de la extensión de esta. Se registraron características clínicas del paciente y del procedimiento. Todos los pacientes fueron seguidos clínicamente durante la internación, al alta y en forma telefónica hasta una semana después de este.

\section{PUNCIÓN CUBITAL Y PROCEDIMIENTO}

La punción se realizó en todos los casos en miembro superior derecho, con el brazo extendido al costado de cuerpo, sobre una superficie plana. En tercio distal del antebrazo se colocó una almohadilla de tal forma que se realizaba extensión de la mano con leve desviación radial, lo que eleva la zona de punción y rectificando la arteria cubital en la zona de pliegue de muñeca. Posteriormente se aplicó subcutáneo de 0,5 a $1 \mathrm{cc}$ de lidocaína al $2 \%$ sin epinefrina dentro de los $2 \mathrm{~cm}$ proximales al hueso pisiforme sobre un segmento de la arteria palpable usando el set de punción e introductor radial hidrofílico Terumo (Figura 1). Con técnica de Seldinger se punzó la arteria cubital. Los introductores utilizados fueron 5 o 6 french $(\mathrm{Fr})$. La canulación arterial corona Figura 1. Técnica de fijación de

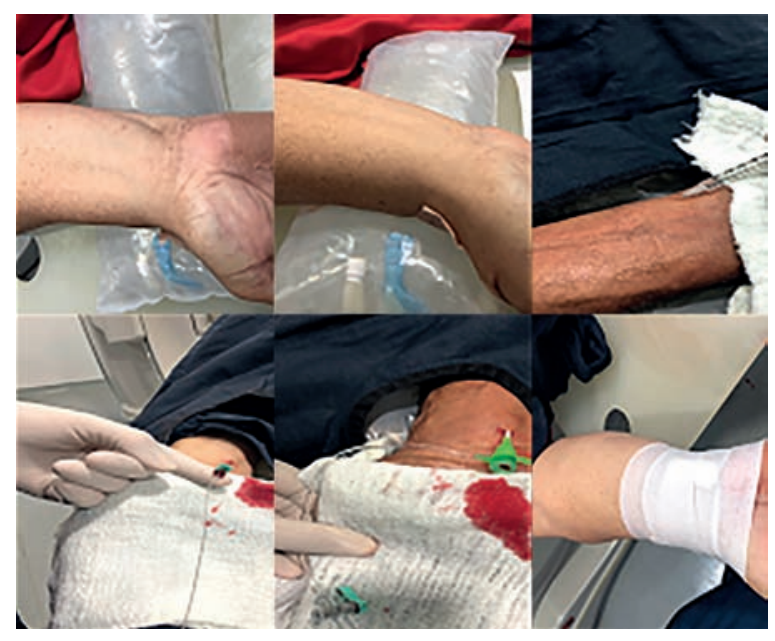

Figura 1. Técnica de fijación de miembro superior derecho y sitio para realizar punción de arteria cubital.

miembro superior derecho y sitio para realizar punción de arteria cubital. ria selectiva para diagnóstico se realizó con catéteres TIGER (TERUMO, Japón), TRAP 3.5 (MEDTRONIC, EE.UU.) y ULTIMATE (5 Fr) (MERITMEDICAL, EE.UU.); para el aortograma se utilizó pig tail (5 Fr), para los procedimientos terapéuticos (angioplastias) se utilizaron catéteres $(6 \mathrm{Fr})$ de la preferencia del operador según la arteria a tratar (VL, JL, EBU, FL, JR). Todos los pacientes recibieron $5000 \mathrm{U}$ de heparina IV al inicio del procedimiento y se completó la dosis hasta alcanzar $100 \mathrm{U} / \mathrm{kg}$ en caso de angioplastia. Todos los introductores fueron retirados inmediatamente terminado el procedimiento aplicando presión sobre el sitio de punción con vendaje compresivo por una hora, posterior a ella se disminuyó al mínimo la presión sobre la zona y 5 horas después se retiró totalmente. El alta de los pacientes se programó según el diagnóstico de ingreso, se le realizó seguimiento hasta el alta y telefónicamente a la semana.

\section{RESULTADOS}

De un total de 1020 pacientes sometidos a procedimientos coronarios, 60 fueron elegidos para realizar acceso por vía cubital como acceso primario (5,8\%). La realización del estudio se dio en diferentes escenarios clínicos: síndromes coronarios agudos con supradesnivel del segmento $S T$, angina inestable, pacientes ambulatorios programados para cateterismo cardíaco. Las características clínicas de los pacientes estudiados se observan en la Tabla 3. Se evidencia en ellos múltiples comorbilidades con elevado riesgo cardiovascular. En todos los pacientes la vía de acceso primaria fue la vía cubital derecha. En 57 pacientes se realizó angiografía diagnóstica (95\%), en $2(3.3 \%)$ solo procedimiento terapéutico programado y en 1 (1.6\%) la punción no fue exitosa. Se realizó aortograma en 11 pacientes (18\%), en 14 se realizó angioplastia coronaria (ATC), a 20 (33\%) se les practicó más de un procedimiento por la misma vía (Tabla 3). Todas las inyecciones fueron selectivas y se realizaron con catéteres TIGER, ULTIMATE o TRAP y esto dependía del centro. No se requirió cambio de catéteres para completar el estudio, no se utilizaron catéteres adicionales a los descritos para los diagnósticos, para los aortogramas pig tail fue el catéter seleccionado, con el que se completó el estudio sin complicaciones. En 14 pacientes (23\%) se realizó procedimiento terapéuticos coronario. de estas angioplastia fueron electiva en 9 pacien- 
TABLA 3. Factores de riesgo.

\begin{tabular}{|l|c|c|}
\hline Características & N pacientes & $\%$ \\
\hline Sexo (F) & 25 & 41 \\
\hline Sexo (M) & 35 & 59 \\
\hline Mayores de 75 años & 5 & 8,3 \\
\hline Edad promedio & 63,3 & \\
\hline Hipertensos & 55 & 91 \\
\hline Diabéticos & 21 & 35 \\
\hline Tabaquismo & 21 & 35 \\
\hline Dislipemia & 44 & 73 \\
\hline Infarto agudo de miocardio previo & 11 & 18 \\
\hline Angioplastia coronaria previa & 10 & 16,6 \\
\hline Cirugía de revascularización coronaria previa & 1 & 1,6 \\
\hline Insuficiencia renal crónica & 8 & 13,3 \\
\hline
\end{tabular}

TABLA 4. ATC electiva vs. urgencia.

\begin{tabular}{|c|c|c|}
\hline Tipo de ATC & Éxito & Mortalidad \\
\hline Electiva & $9 / 9(100 \%)$ & $0 / 9(0 \%)$ \\
\hline Urgencia & $5 / 5(100 \%)$ & $0 / 5(0 \%)$ \\
\hline Total & $14 / 14(100 \%)$ & $0 / 14(0 \%)$ \\
\hline
\end{tabular}

tes (14\%), 2 pct de estos se les realizo la ATC como procedimiento único, angioplastia coronaria de urgencia por síndrome coronario agudo con supra desnivel del ST se realizaron en 5 pacientes (9\%), la intervención de más de dos vasos se realizó en 2 pacientes (Figura 2).

Se realizó angioplastia a un vaso en 11 pacientes (18\%), angioplastia a dos vasos en $1(1.6 \%)$, angioplastia a múltiples vasos (más de dos vasos) en 2 (3.2\%), con tasas de éxito del $100 \%$ y $0 \%$ de mortalidad hasta el seguimiento realizado; los vasos tratados así como número y porcentajes se describen en la Tabla 4. Para las angioplastias se utilizaron catéteres BL (TERUMO, Japón), Judkins left (JL) (Boston Scientific, procedentes de México), EBU (Medtronic, EE.UU.), $\mathrm{Fe}$ moral left (FL) (Boston Scientific, procedentes de Holanda), Judkins right (JR) (Boston Scientific procedentes de México), IKARI (TERUMO, Japón). Estos fueron elegidos según arteria a tratar y a consideración de operador para soporte requerido durante el procedimiento los intercambios de catéteres se realizaron sobre guía J 0,35 x 300 cm sin complicaciones. En un paciente la punción no fue exitosa por ser prolongada (más de dos intento de punción de la arteria cubital), lo que obligó a cross over a vía radial ipsilateral (1,6\%). Un paciente $(1,6 \%)$ presentó complicación del sitio de punción por la presencia de hematoma leve $(1,6 \%)$ posterior al estudio, esto secundario a una mala técnica de compresión del sitio de punción por parte del personal a cargo de retirar el introductor, por lo que se realizó capacitación al personal y no se evidenció en el resto de pacientes este tipo de complicaciones hasta el alta y hasta 1 semana posterior al estudio. El 98,3\% de los procedimientos fueron exitosos. Durante el estudio se notó después del caso \#15 el mejoramiento de la técnica de punción de arteria cubital siendo esta mucho más rápida y segura. Ningún paciente presentó espasmo clínico de la arteria cubital, ningún paciente presento alteraciones nerviosas periféricas relacionadas con la punción cubital durante el tiempo se seguimiento.

En cuanto a nuestros objetivos planteados al realizar este estudio, con respecto a la factibilidad del acceso cubital como vía primaria encontramos que los resultados, con tasa de éxito del 98,3\%, corroboran que el acceso cubital es una vía elegible como acceso primario para realizar estudios hemodinámicos.

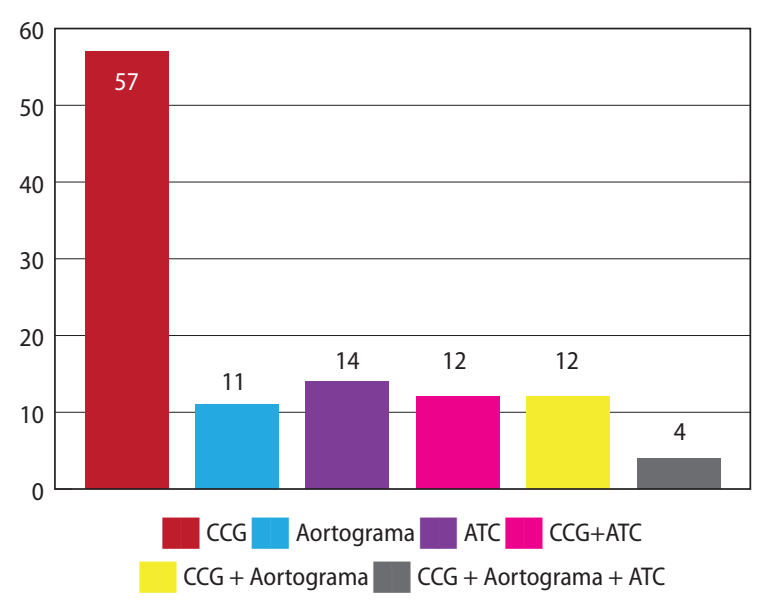

Figura 2. Procedimientos realizados. CCG: cinecoronariografía. ATC: angioplastia coronaria.

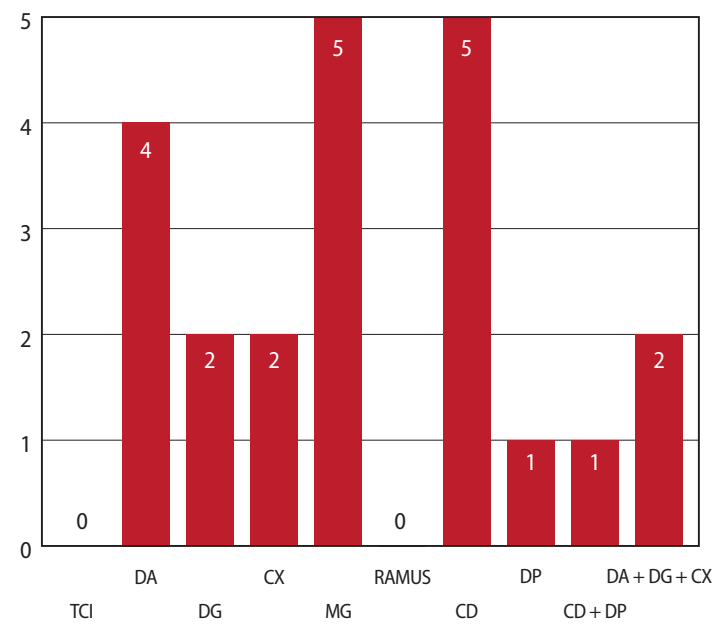

Figura 3. Arterias tratadas. TCl: tronco de coronaria izquierda. DA: descendente anterior. DG: diagonal. CX: circunfleja. MG: margina. CD: coronaria derecha. $D P$ : descendente posterior.

El segundo objetivo, que era determinar la seguridad para realizar el estudio por este acceso, también arrojó resultados favorables, con una tasa de éxito para completar el estudio cercanos al $100 \%$, con baja tasa de cross over $(1,6 \%), 0 \%$ de complicaciones durante el estudio, 1,6\% de complicaciones posterior a este en zona de punción y sin complicaciones nerviosas de la zona de punción, además de poder completar el estudio con catéter único para la arteriografía coronaria. Adicional a esto se evidenció la versatilidad para realizar diferentes estudios angiográficos por la misma vía durante el mismo procedimiento (cateterismo cardíaco, aortograma, angioplastia coronaria), sin presencia de vasoespasmo asociado al intercambio de catéteres.

\section{DISCUSIÓN}

El abordaje por el antebrazo para estudios diagnósticos y terapéuticos coronarios se ha convertido en el preferido del especialista por la comodidad para su ejecución además del confort y seguridad para el paciente ${ }^{7,8}$. La realización de los estudios angiograficos coronarios diagnósticos y terapéuticos en nuestros centros es del $97 \%$ de los casos por vía radial para estudios coronarios, lo cual es similar a la tendencia 
mundial y a lo encontrado en la bibliografía consultada. El porcentaje de estudios por vía cubital correspondió al 5,8\% del total. Durante la realización de este estudio se presentó un caso de cross over a vía radial por punción prolongada y un caso de hematoma en zona de punción secundario a mala técnica para la colocación del vendaje compresivo, lo cual es estadísticamente comparable con la literatura para otros accesos en antebrazos ${ }^{8,9}$. En catorce de los pacientes se realizó angioplastia; 5 de estos fueron pacientes con SCASEST, incluido uno en shock cardiogénico, y 9 correspondían a angina inestable quienes se sometieron electivamente angioplastia; en dos de ellos se decidió realizar angioplastia a múltiples vasos, que no tuvo complicaciones y con tasas de éxito y mortalidad comparables a las reportadas al ser realizadas por otros abordajes. Además se realizaron aortogramas en 11 pacientes, realizando intercambio de catéteres sin complicaciones según la elección del operador. Es de resaltar que la menor presencia de receptores alfa en la arteria cubital asociado a su mayor diámetro en comparación a la arteria radial disminuye la presencia de espasmos arteriales y mejora la posibilidad de punción y desarrollo exitoso del estudio ${ }^{10}$. La experiencia previa de la técnica de punción radial habitual tiene incidencia directa la curva aprendizaje para la punción exitosa de la vía cubital, esto asociado a una adecuada técnica de punción y posicionamiento de la extremidad lleva a que se requiera un menor número de pacientes para dominar la técnica de este acceso, en nuestra experiencia notamos que posterior a las 15 punciones la técnica mejoro en tiempo y se-

\section{BIBLIOGRAFÍA}

1. Feldman DN, Swaminathan RV, Kaltenbach LA, et al. Adopción del acceso radial y comparación de los resultados con el acceso femoral en la intervención coronariapercutánea: un informeactualizado del Registro Nacional de Datos Cardiovasculares (2007-2012). Circulation 2013;127:2295306

2. KiemeneijF,Laarman GJ. Percutaneoustransradial artery approach forcoronary Palmaz-Schatz stent implantation. Am Heart J 1994;128:167-174.

3. Terashima M, Meguro T, Takeda H, Endoh N, et al. Percutaneous ulnar artery approach for coronary angiography: a preliminary report in nine patients. Catheter Cardiovasc Interv 2001;53:410-4.

4. Méndez M, Pérez O. Acceso por vía cubital como alternativa al acceso radial para diagnóstico e intervenciones coronarias. Rev Chil Cardiol 2011;30:16-9.

5. Li YZ, Zhou YJ, Zhao YX, et al. Safety and efficacy of transulnar approach forcoronary/angiography andintervention. Chin Med J 2010;123:1774-9.

6. Aptecar E, Pernes JM, Chabane-Chaouch M, et al. Transulnarversus trans- guridad, con una muy baja tasa de complicaciones. Con ello que afianza la factibilidad y la seguridad del acceso por vía cubital derecha como elección primaria para realizar estudios coronarios tanto diagnósticos como terapéuticos y angiograficos de otro tipo.

\section{LIMITACIONES}

Al realizar este estudio nos encontramos con limitaciones por ser un muestra pequeña, además de ser un estudio observacional no aleatorizado. Otra limitante se encuentra en el seguimiento a largo plazo de los pacientes en los que se utilizó la vía cubital como acceso primario, lo que no permite demostrar con imágenes (Doppler u otro mecanismo) la permeabilidad del vaso más allá del tiempo de seguimiento de una semana. Otra limitación fue la elección de la vía cubital en antebrazo derecho sin evaluar el acceso por esta vía en miembro superior izquierdo.

\section{CONCLUSIÓN}

De acuerdo a los resultados obtenidos, el acceso cubital como vía primaria es factible y seguro para la realización de estudios coronarios ya sean diagnósticos o terapéuticos coronarios. Además, se pueden realizar otros tipos de estudios angiográficos y múltiples procedimientos durante el mismo acto con excelentes tasas de éxito, bajas tasas de complicaciones, independientemente del contexto clínico del paciente. radial artery approach for coronaryangioplasty: the PCVI-CUBAstudy. Catheter Cardiovasc Interv. 2006;67:711-20.

7. Caputo RP, Tremmel JA, Rao S, et al. Acceso arterial transradial para procedimientos coronarios y periféricos: resumen ejecutivo del Comité Transradial del SCAl. Cathet y Cardiovasc Interv 2011;78:823-39.

8. WuCJ, LOPH, Chang KC. Transradial coronaryangiographyand angioplasty in Chinese patients. Send to Cathet Cardiovasc Diagn 1997;40(2):15963.

9. Agostoni P, Biondi-Zoccai GG, de Benedictis MLRadial versus femoral approach for percutaneous coronary diagnostic and interventional procedures: Systematic overview and meta-analysis of randomized trials. J Am Coll Cardiol 2004 Jul 21;44(2):349-56.

10. Roberts EB, Palmer N, Perry RA. Acceso transulnar para la anagiografía coronaria y la intervención: una revisión temprana para guiar la investigación y la práctica clínica. J Invasive Cardiol. 2007;19:83-7. 